\title{
A developmental evaluation of the role of word shape in word recognition
}

\author{
ALICE F. HEALY \\ University of Colorado, Boulder, Colorado \\ and \\ THOMAS F. CUNNINGHAM \\ St. Lawrence University, Canton, New York
}

\begin{abstract}
Fourth-and seventh-grade children and college-age adults proofread passages typed either in lowercase or in all capital letters. Words were misspelled by deleting one of four letters, s, c, k, or $p$, that have similar features in lowercase and uppercase. Proofreading errors decreased with age and increasing reading ability, but all of the subjects were sensitive to changes in word shapethey missed more words with deletions of 8 or $c$ than $k$ or $p$ in the lowercase passage but not in the all-capitals passage. These findings indicate that word shape is an important variable in recognizing familiar words, even for young readers.
\end{abstract}

Early in the history of psychology, it was proposed that global word-shape information, or information concerning supraletter features (i.e., features involving more than one letter or the relationship among letters), plays an important role in word recognition (see, e.g., Cattell, 1886; Erdmann \& Dodge, 1898). More recently, however, influential models of word processing have been proposed that do not include supraletter feature detection (see, e.g., McClelland \& Rumelhart, 1981). Furthermore, recent experimental investigations have suggested that word rec-

\footnotetext{
The two authors contributed equally to this research; order of authorship was determined arbitrarily. Parts of this project were conducted at St. Lawrence University in Canton, New York, and at the University of Colorado, Boulder. A brief report of this work was presented at the 27th Annual Meeting of the Psychonomic Society in New Orleans, November 14, 1986. The research was supported in part by special research funds provided through a Faculty Research Award and a Student Research Award from St. Lawrence University, by National Science Foundation Grant BNS80-25020 and Army Research Institute Contract MDA903-90-K-0066 to the Institute of Cognitive Science at the University of Colorado, and by a Council on Research and Creative Work research grant-in-aid from the University of Colorado. We wish to thank the students and teachers in the central school systems of Canton and Ogdensburg, New York, who participated in the present experiment or in preliminary pilot work. Our project was facilitated by the splendid cooperation given by Kathy Curro, Principal of Banford Elementary School in Canton, and by Betty Mallot, Principal of the Kennedy Elementary School in Ogdensburg. We wish especially to thank Marie Truax, Assistant Principal of the Ogdensburg Junior High School, for her extensive help and encouragement. Thanks are also due to Grant Sinclair for his help in testing the college students, to Rebecca L. Willits for her work in constructing the experimental materials, to Lisa Kunitz, Whitney Bruce, and Bill Marmie for their help in data tabulation and analysis, and to David Peterzell, Guy van Orden, Margaret Jean Intons-Peterson, Nelson Cowan, and two anonymous reviewers for their helpful cormments about this research. Requests for reprints should be sent either to Alice F. Healy, Department of Psychology, University of Colorado, Campus Box 345, Boulder, CO 80309-0345 or to Thomas F. Cunningham, Department of Psychology, St. Lawrence University, Canton, New York 13617.
}

ognition is mediated by the identification of letter units and that there is no need to postulate word shape as making an independent contribution to word identification (see, e.g., Besner, 1989; Oden, 1984; Paap, Newsome, \& Noel, 1984). However, other new studies have suggested that word shape may be useful in word recognition if it is coupled with the presence of other cues, such as other orthographic features (see Walker, 1987) and syntactic and semantic features from the surrounding prose context (see L. R. Haber, R. N. Haber, \& Furlin, 1983).

One experimental task that has been used to study the effects of word shape on word recognition is proofreading for misspellings. This task is high in ecological validity because the subject engages in reading continuous text, which provides a prose context similar to that in normal reading. Another positive characteristic is that in proofreading, the subject can make use of parafoveal information, which has been shown to be particularly useful in guiding eye movements during the reading of text (see, e.g., Hadley \& Healy, 1991; O'Regan, 1979; Rayner, 1979; Rayner, McConkie, \& Zola, 1980). The proofreading task has yielded evidence for the importance of word-shape information in word processing. For example, R. N. Haber and Schindler (1981) and Monk and Hulme (1983) created misspellings by replacing one letter in a word with another letter so that word shape, as defined by the pattern of ascending, descending, and neutral letters, was either altered or maintained. The number of proofreading errors was substantially larger, especially in short words, when word shape was not changed by the substitution (but see Abramovici, 1983). These results seem to imply that word shape influences word recognition, but they do permit an altemative interpretation. Specifically, as argued by Monk and Hulme (1983), Oden (1984), and Paap et al. (1984), the effects of word shape in these experiments could be localized at the let- 
ter level rather than at the word level because the substitutions that maintained shape undoubtedly involved more confusable (i.e., less discriminable) letters than did the substitutions that changed shape (see also Garner, 1981, and McClelland, 1977, for discussions of the relationship between word shape and letter shape). Indeed, it was shown in other proofreading experiments that whether or not word shape was maintained by the substitution had less influence on proofreading error rate than did the visual similarity of the original letter to the one that replaced it (see Healy \& Drewnowski, 1983, Experiment 2; Paap et al., 1984, Experiment 1).

The results with the substitution proofreading procedure are thus ambiguous. Another proofreading procedure, however, does not lead to such ambiguity. In this procedure, originated by Corcoran (1967) and later developed by Monk and Hulme (1983), letters are deleted from words rather than replaced by other letters (e.g., latest is misspelled as latet). With this procedure, Monk and Hulme also showed that subjects in proofreading were more likely to miss misspellings that maintained word shape than those that changed shape. But in this case, the effect of changes in word shape cannot be localized at the letter level because the letter that was manipulated was deleted and was therefore not available to influence the letter identification processes.

More specifically, in the study by Monk and Hulme (1983) college-age subjects proofread a passage of conventionally printed text that contained mispellings, some of which were created by deleting a consonant from one of two sets: ascenders $(b, d, f, h, k, l, t)$ and neutral letters $(c, m, n, r, s, v, w)$. Subjects made significantly more proofreading errors when a neutral letter was deleted than when an ascender was deleted. To control for nonvisual factors that might have contributed to the difference between misspellings involving the two letter sets, a second experiment was conducted in which the text that was proofread was printed with half of the letters in lowercase and half in uppercase. In this control condition, the effects of letter set were eliminated, presumably because word shape was changed for all misspelled words, not just for those involving deleted ascending letters.

In the present study, we sought to confirm and extend the findings of Monk and Hulme (1983) by modifying their procedure in three crucial respects. First, we changed the control or baseline condition to involve all capital letters rather than mixtures of lowercase and uppercase characters. Because the definition of word shape we were using, with respect to ascending, descending, and neutral letters, applies only to lowercase print, any effects of word shape should be eliminated with pure uppercase printing, as well as with mixed case printing (see Healy \& Drewnowski, 1983, Paap et al., 1984, and Underwood $\&$ Bargh, 1982, for previous uses of the pure uppercase control). In addition, pure uppercase printing is not subject to the criticism of case mixing that it is very unnatural and hence might disrupt the normal reading processes (see, e.g., Oden, 1984, Underwood \& Bargh, 1982).
The second change we introduced into Monk and Hulme's procedure concerned the sets of letters that were deleted. We employed only four different letters in creating the misspellings: $s, c, k$, and $p$. We selected these letters because each of them has essentially the same features when printed in lowercase and uppercase (e.g., compare $s$ and $S$ with $b$ and $B$ ). Nevertheless, in lowercase, these letters are placed into three different shape categories, following the letter classification scheme originally proposed by Bouma (1971): ascending (k), descending (p), or neutral ( $s$ and $c)$. In contrast, in uppercase, no such distinctions are made, because the letters are all of the same height. In uppercase print, deletion of any one of these letters should not affect word shape, whereas in lowercase, word shape is unaffected only when the neutral letters $s$ and $c$ are deleted. When the letters $p$ and $k$ are deleted in lowercase, word shape is altered. Therefore, if word shape does influence word-recognition processes, we should find an interaction of letter case and type of letter deletion on proofreading errors. We should emphasize that we are predicting an interaction, but we cannot predict, on the basis of our hypothesis about word shape, whether there will also be main effects of letter case and type of letter deletion. Because factors such as the frequency and length of a test word and the location of the deleted letter in the test word undoubtedly affect the tendency to make proofreading errors on that word, we cannot predict in advance whether test words with $p$ or $k$ deleted will show more or fewer errors for the uppercase baseline than will test words with $\mathrm{s}$ or $\mathrm{c}$ deleted. (Indeed, the difference between $p$ and $k$ test words and $s$ and $c$ test words in uppercase may vary with the specific set of words employed.) Likewise, because the decreased size of lowercase print relative to uppercase print may disturb proofreading accuracy, whereas the greater familiarity of lowercase print relative to uppercase print may aid proofreading accuracy, we cannot predict in advance whether there will be an increase, a decrease, or no difference in proofreading errors on lowercase relative to uppercase for the neutral $s$ and $c$ test words. However, because word frequency, word length, and the location of the deleted letter in the test words are the same for lowercase and uppercase, we are able to predict the form of the interaction between letter case and type of letter deletion: Word shape is changed with the letter deletion only for the $p$ and $k$ test words in lowercase; hence, any increase in proofreading errors for lowercase relative to uppercase found for the netural $s$ and $c$ test words should be reduced or reversed for the $\mathrm{p}$ and $\mathrm{k}$ test words (or any decrease in proofreading errors for lowercase relative to uppercase found for the neutral $s$ and $c$ test words should be enhanced or exaggerated for the $\mathrm{p}$ and $\mathrm{k}$ test words). Alternatively, any increase in proofreading errors found for the uppercase baseline for $p$ and $k$ test words relative to $s$ and $c$ test words should be reduced or reversed for lowercase (or any decrease in proofreading errors found for the uppercase baseline for $p$ and $k$ test words relative to $s$ and $c$ test words should be enhanced or exaggerated for lower- 
case). Because the deleted letters have similar features in the two typecases, it would be difficult to attribute any such interaction we find in the pattern of proofreading errors to differences in features other than those defining word shape.

The third important new aspect of our procedure involved the subjects we tested. We employed children of various ages and reading abilities in Experiment 1 and college-age adults in Experiment 2 so that we could examine the relationship between age, reading level, and word shape. On the basis of earlier work suggesting a developmental increase in the size of the processing units used in reading (e.g., Cunningham, Healy, Kanengiser, Chizzick, \& Willitts, 1988; Drewnowski, 1978, 1981), we would predict that the use of word shape would increase both as a function of the subjects' age and as a function of reading level within age groups. However, other studies in the literature lead to alternative predictions.

A number of previous studies that have emphasized the importance of visual processing and word shape in children's word recognition have employed a picture-word interference task similar to the Stroop (1935) color-word interference task. In this task, the subjects are asked to name as quickly as possible objects represented by line drawings that have a printed sequence of letters (a word or nonword) placed in the center of the picture. The characteristics of the printed sequence are varied to determine the amount of interference in producing a verbal label for the drawing. For example, in Experiment 1 of a tachistoscopic study by Posnansky and Rayner (1977), the picture-naming latencies of children in the first, third, and sixth grades and of college students were compared in nonword conditions that either preserved or altered end letters and/or the word shape of the actual word label. It was found that preservation of the end letters and the word shape in nonwords speeded the naming of pictures. The ability to make use of these word characteristics did not vary with the age of the subjects. Subsequent research by Guttentag (1981) explored individual differences in the use of word shape in children's automatic word processing as a function of age (third and fifth graders) and reading-ability level by using a nontachistoscopic version of the picture-word interference task. As found previously by Posnansky and Rayner, the effect of word shape did not vary by age of the children, but in this case, it did interact with reading-ability level; children who were poor at using spelling-sound correspondence rules were affected by the manipulation of word shape, whereas good readers were not.

Based on the developmental studies with the pictureword interference task, we expect that our subjects will improve their proofreading skill with age but that the relative contribution of word shape to the recognition of words will not change over age groups. However, we may find that word shape contributes more to children's word recognition in poor than in good readers.

\section{EXPERIMENT 1}

We employed fourth- and seventh-grade children in Experiment 1.

\section{Method}

Subjects. We used 120 subjects: 48 children from the fourth grade and 72 children from the seventh grade of a public elementary school system in northern New York State. The children in the fourth and seventh grades were divided evenly into three reading-level groups based on the raw scores they had obtained at the end of the previous school year on two different forms (Grade 3-Form $R$ and Grade 6-Form E) of the Reading Test for New York State Elementary Schools $(1982,1983)$. The reading test, also commonly known as the Degrees of Reading Power Test, consists of a series of nonfiction prose passages of increasing order of difficulty and is given at the end of the school year to third- and sixth-grade students in New York State. Raw scores on the reading test may range from 0 to 56 and from 0 to 77 at lower and higher grade levels, respectively. Descriptive statistics for the reading-ability groups of the fourth and seventh grades with respect to the raw scores obtained on the reading test are given in Table 1 . In addition, we obtained normative data from the New York State Department of Education for the cumulative percentage of students who obtained different raw scores on the two forms of the test in 1984. The sample sizes were 210,832 students and 220,814 students in the third and sixth grades, respectively. Based on these data, the ranges in percentile ranks for the low-, medium-, and high-reading-ability groups in our fourth-grade sample were approximately 11-45, 48-71, and 77-97, whereas the ranges in percentile ranks for the three corresponding reading-ability groups of the seventh grade were approximately $13-48,51-77$, and $80-100$. The average chronological ages and standard deviations ( $S D$ s) for the children in the fourth and seventh grades were 10.06 years $(S D=.68)$ and 12.96 years $(S D=.54)$, respectively.

Materials. Two 656-word passages were created. The passages contained each of the following four types of test words: words with a single $s, c, k$, or $p .^{1}$ Each passage had a total of 80 test words, 20 of each type. The test words were selected with the use of the following constraints: (1) All test words were content words, not function words; (2) all words included the letter a, which appeared before the critical letter that was deleted (this constraint was employed so that we could use this passage again for another purpose); (3) there was only one letter a per word; (4) the critical letter never appeared as the first or last letter of a test word, and the test word itself did not appear as the first word of a sentence or the first or last word

Table 1

Descriptive Statistics for the Raw Scores Obtained on the Reading Test by the Low-, Medium-, and High-Reading-Ability Groups of Grades 4 and 7 in Experiment 1

\begin{tabular}{lrrrr}
\hline $\begin{array}{l}\text { Reading } \\
\text { Ability }\end{array}$ & $n$ & Range & $M$ & $S D$ \\
\hline & & Grade 4 & & \\
Low & 16 & $20-36$ & 31.63 & 4.56 \\
Medium & 16 & $37-44$ & 39.69 & 2.06 \\
High & 16 & $46-53$ & 48.69 & 2.77 \\
& & Grade 7 & & \\
Low & 24 & $42-59$ & 54.04 & 4.79 \\
Medium & 24 & $60-68$ & 64.58 & 2.84 \\
High & 24 & $69-77$ & 72.63 & 2.58 \\
\hline
\end{tabular}


of a typed line; (5) a test word was always four or more letters in length and was not a name or proper noun; and (6) each test word was chosen so that when the critical letter $(s, c, k$, or $p)$ was deleted, another correctly spelled word was not formed (with one exception, carpet was misspelled as the relatively rare word caret).

The two passages differed only with respect to the test words that were misspelled. A given test word was correctly spelled in one passage and misspelled in the other passage by deleting the critical letter; in each passage, half of the test words of each type were misspelled. The words misspelled in each passage were randomly selected from the four types of test words. Thus, each passage contained a different set of 40 test words that had been misspelled, 10 of each word type. The Appendix provides a listing of each test word for each word type and passage. There were also two different versions of each passage, one with all uppercase letters and the other with standard capitalization so that all test words appeared in lowercase. Each passage version was typed in Courier 10 pitch with double spacing on two sheets of paper.

A multiple-choice reading comprehension test was constructed to include nine questions, each with three alternative answer choices. Across questions, the correct answer occurred four times in position A, twice in B, and three times in C. All questions could be easily answered on the basis of the information provided in the passage.

Two versions of a 41-word spelling test were also constructed, one for each passage. Each of the misspelled test words in the corresponding passage was included both spelled correctly and misspelled with the critical letter deleted. In addition, the word home, correctly spelled and misspelled as $h m e$, was included as an initial practice item. Each word was typed on a separate line, with the word's two different spellings in different vertical columns. The words occurred on the spelling test in the same order in which they occurred in the passage. The correctly spelled versions of 19 of the 40 test words were in one column; the remaining 21 correctly spelled test words were in the other column.

Procedure. Each subject was given a standard set of instructions and both versions (lowercase and uppercase) of one of the two passages to read. Each child within a reading-ability group was randomly assigned to one of the two passages and one of the two sequences in which the two versions were to be read. An equal number of children within each reading-ability group received each passage with the order of presentation of passage versions counterbalanced across subjects. To ensure that the subjects read the passages for meaning, all of the subjects were told at the beginning of the study that they would be required to answer multiple-choice questions pertaining to the content of the passage after they completed the proofreading task.

The children were tested in a classroom in groups of 10 to 15 individuals. All of the subjects had completed another task prior to beginning the proofreading task. The subjects were asked to read at their normal reading speed and to circle the misspelied words with their pencils. They were also told to read the two versions of the passage before stopping to rest. The subjects were not informed in advance that they would be reading the same passage in two different formats. It was emphasized that the subjects should not return to an earlier part of the passage if at any time they realized that they had missed circling a misspelled word. They were told that they were not expected to catch every misspelled word and that they should not slow down in order to do so. Emphasis was placed on the questions at the end of the passage, which the subjects were told to read for meaning. The children were asked to raise their hands when they were finished reading the passage versions so that they could be timed.

After reading the two passage versions, the subjects completed the remaining two sections of the experiment, which were the comprehension test followed by the spelling test. Neither of these tests was timed. In both tests, the subjects were instructed to circle the correct alternative. The children completed the spelling test in a separate session that occurred within 2 weeks of the original test session.

\section{Results}

Proofreading errors. The results of the proofreading task are summarized in Table 2 with respect to error percentages as a function of subject age and reading-ability level, letter case (lower or upper), and type of letter deletion (neutral $-s$ and $c-$ or ascender/descender $-k$ and $p$ ).

A mixed factor analysis of variance (ANOVA) was conducted to assess the relationship among the betweensubject variables of age and reading-ability level (low, medium, high) and the within-subject variables of letter case and type of letter deletion.

Significant main effects were obtained for age $[F(1,114)$ $\left.=30.98, M S_{\mathrm{c}}=755.42, p<.001\right]$ and reading-ability level $\left[F(2,114)=12.10, M S_{\mathrm{e}}=755.42, p<.001\right]$, indicating that the seventh-grade subjects made fewer proofreading errors overall than did the fourth-graders and that higher reading-ability-level children in both grades made fewer proofreading errors than did children of lower reading ability. There was also a main effect caused by letter case $\left[F(1,114)=4.27, M S_{c}=68.80, p=.041\right]$. More proofreading errors were made when the letters were in uppercase $(M=26.21 \%)$ than when they were in lowercase $(M=24.81 \%)$. The only remaining significant effect, and the one of primary interest, was a significant interaction between letter case and type of letter deletion $\left[F(1,114)=30.12, M S_{\mathrm{e}}=50.36, p<.001\right]$. There was a difference in the pattern of proofreading errors made between deleting $s$ and $c$ and deleting $k$ and $p$ to form the misspelled test words. As predicted, although in uppercase print more proofreading errors were made on the $p$ and $k$ test words than on the $s$ and $c$ test words, in lowercase print more proofreading errors were made on the $s$ and $c$ test words that retained word shape than on the $k$ and $p$ test words that altered shape. The overall pattern of errors reflected in this interaction did not vary as a function of age or reading-ability level. The percentage of proofreading errors was $26.79 \%$ for $s$ and $c$ words and $22.83 \%$ for $k$ and $p$ words in lowercase print, whereas the comparable figures in uppercase print were $24.67 \%$ and $27.75 \%$, respectively. We used the least significant

Table 2

Proofreading Error Percentages as a Function of Subject Age and Reading Ability (for Fourth and Seventh Graders), Letter Case, and Type of Letter Deletion in Experiments 1 and 2

\begin{tabular}{|c|c|c|c|c|}
\hline \multirow[b]{3}{*}{ Age Level } & \multicolumn{4}{|c|}{ Letter Case } \\
\hline & \multicolumn{2}{|c|}{ Lowercase } & \multicolumn{2}{|c|}{ Uppercase } \\
\hline & $s$ and $c$ & $k$ and $p$ & $\mathrm{~S}$ and $\mathrm{C}$ & $\mathbf{K}$ and $\mathbf{P}$ \\
\hline \multicolumn{5}{|c|}{ Experiment 1} \\
\hline \multicolumn{5}{|l|}{ Grade 4} \\
\hline Low reading ability & 41.2 & 39.4 & 42.5 & 48.7 \\
\hline Medium reading ability & 30.9 & 29.1 & 27.8 & 38.1 \\
\hline High reading ability & 29.7 & 26.2 & 26.9 & 28.1 \\
\hline \multicolumn{5}{|l|}{ Grade 7} \\
\hline Low reading ability & 30.4 & 24.2 & 27.1 & 26.9 \\
\hline Medium reading ability & 21.5 & 17.5 & 21.2 & 22.3 \\
\hline High reading ability & 14.2 & 9.4 & 10.2 & 12.9 \\
\hline \multicolumn{5}{|c|}{ Experiment 2} \\
\hline College & 15.2 & 12.6 & 12.4 & 16.5 \\
\hline
\end{tabular}


difference $(L S D)$ test $(\alpha=.05, L S D=1.81)$ to examine the significance of our independent planned comparisons. The percentage of errors was significantly greater for $s$ and $c$ test words than for $k$ and $p$ test words in lowercase, whereas the opposite pattern was significant in uppercase. Furthermore, there was a significant increase in proofreading errors for lowercase relative to uppercase with the neutral $s$ and $c$ test words, whereas the opposite pattern was significant with the $k$ and $p$ test words.

Proofreading times. The mean time to proofread the two passage versions is shown in Table 3 as a function of subject age and reading-ability level.

A two-factor (age and reading-ability level) ANOVA was conducted on the proofreading times. The seventh graders had shorter times on the average than did the fourth graders $\left[F(1,113)=36.41, M S_{\mathrm{c}}=38,033.89\right.$, $p<.001]$, and the high-reading-ability children generally had shorter times than the children of low and medium reading ability $\left[F(2,113)=5.35, M S_{\mathrm{e}}=38,033.89, p=\right.$ $.006]$. The interaction between age and reading ability was not significant $[F(2,113)<1]$.

Comprehension test scores. The mean frequency of correct responses on the nine-item comprehension test is shown in Table 3 as a function of subject age and reading ability.

As in the analysis of proofreading times, a two-factor ANOVA was conducted on the comprehension test scores, with the factors comprising age and reading-ability level. Although the scores of the seventh graders were somewhat higher than those of the fourth graders, the main effect of age was not statistically significant $[F(1,114)=$ 2.62, $M S_{\mathrm{e}}=0.85, p=.108$ ]. Performance did, however, reliably increase as a function of reading-ability level $\left[F(2,114)=7.66, M S_{\varepsilon}=0.85, p<.001\right]$. The interaction of age and reading-ability level was not significant $[F(2,114)<1]$.

Spelling test. Performance on the spelling test was quite high for all subjects. The mean percentages of correct responses (and standard errors of the mean) were $97.3 \%$ $(0.5 \%)$ and $99.2 \%(0.3 \%)$ for the fourth graders and

Table 3

Mean Proofreading Time (in Seconds) and Mean Frequency of Correct Responses on the Comprehension Test as a Function of Subject Age and Reading Ability (for Fourth and Seventh Graders) in Experiments 1 and 2

\begin{tabular}{lcc}
\hline \multicolumn{1}{c}{ Age Level } & $\begin{array}{c}\text { Proofreading } \\
\text { Time }\end{array}$ & $\begin{array}{c}\text { No. Correct } \\
\text { on Comprehension }\end{array}$ \\
\hline Grade 4 & Experiment 1 & \\
Low reading ability & 920 & 7.4 \\
Medium reading ability & 952 & 7.9 \\
High reading ability & 820 & 8.2 \\
Grade 7 & & \\
Low reading ability & 747 & 7.8 \\
Medium reading ability & 699 & 8.1 \\
High reading ability & 587 & 8.6 \\
& Experiment 2 & \\
College & 472 & 8.5 \\
\hline
\end{tabular}

Table 4

Breakdown of Errors on the Spelling Test as a Function of Subject Age and Type of Letter Deletion in Experiments 1 and 2

\begin{tabular}{cccc}
\hline & \multicolumn{2}{c}{ Type of Letter Deletion } & \\
\cline { 2 - 3 } Age Level & S and C & K and P & Total \\
\hline \multirow{4}{*}{ Experiment 1} & \\
Grade 4 & 27 & 26 & 53 \\
Grade 7 & 13 & 10 & 23 \\
& Experiment 2 & & \\
College & 1 & 0 & 1 \\
\hline
\end{tabular}

seventh graders, respectively. Across all subjects, only 76 errors were made in total. The distribution of errors as a function of subject age and type of letter deletion is shown in Table 4. The number of errors made on words with $s$ and $c$ deleted (40) is roughly equivalent to that made on words with $k$ and $p$ deleted (36).

\section{EXPERIMENT 2}

Experiment 2 was like Experiment 1 except that college students rather than elementary school children were employed as subjects.

\section{Method}

Subjects. The subjects were 40 college students enrolled in psychology courses at the University of Colorado, Boulder.

Materials. The materials were the same as those used in Experiment 1 .

Procedure. The procedure was the same as in Experiment 1, except that 16 students were tested individually and the remaining 24 students were tested in a large classroom setting. For college students who were tested individually, the procedure for timing was the same as in Experiment 1. However, the 24 college students who were tested as a group were given a standard starting time and instructed to record the time (available from a large clock in the testing room) when they completed reading the passage versions. Unlike in Experiment 1, the subjects in Experiment 2 completed all tasks, including the spelling test, in one testing session.

\section{Results}

Proofreading errors. The results of the proofreading task are summarized in Table 2 with regard to error percentages as a function of letter case and type of letter deletion. The Appendix also provides a summary of the error frequencies for each test word in each passage as a function of typecase and type of letter deletion.

A repeated measures ANOVA was conducted to assess the relationship between the within-subject variables of letter case and type of letter deletion. In accord with predictions and with the results of Experiment 1, the interaction between letter case and type of letter deletion was significant $\left[F(1,39)=9.70, M S_{\mathrm{e}}=46.97, p=.004\right]$. Based on the $L S D$ test value ( $\alpha=.05, L S D=3.10)$, there were (1) a significant decrease in errors on the $k$ and $p$ test words that changed word shape (and a nonsignificant increase in errors on the $s$ and $c$ test words that maintained word shape) in lowercase relative to the uppercase baseline and (2) significantly fewer errors on the neutral 
$\mathbf{s}$ and $\mathrm{c}$ test words than on the $\mathbf{k}$ and $\mathrm{p}$ test words for the uppercase baseline but a nonsignificant trend in the opposite direction for lowercase.

To determine whether this crucial interaction was generalizable across items (i.e., test words) as well as across subjects, a second ANOVA was conducted with items, rather than subjects, as the random effect. In this analysis, type of letter deletion was a between-item factor and letter case was a within-item factor. The important interaction of these two factors remained significant $[F(1,78)$ $\left.=10.35, M S_{\mathrm{e}}=44.03, p=.002\right]$.

Support for the importance of word shape was, therefore, provided by finding a significant interaction of letter case and type of letter deletion in both the analysis with subjects and that with items as the random effect. An alternative explanation that must be considered for this interaction is that in the context of lowercase words, the encoding of certain letters could include the ascending (e.g., k) or descending (e.g., p) feature. Rather than check word shape, the subjects could check that all of the features in all of the letters of the word were present. If the word was supposed to contain only one ascending (or descending) letter, the absence of that critical feature could be easily detected. In contrast, within most words, the neutral feature (e.g., s or c) is shared with other letters in the word, so the absence of that feature might not be so salient. To test this alternative explanation, a post hoc analysis was conducted on the proofreading error percentages, separating the $k$ and $p$ test words into those with no other letters with the same ascending (or descending) feature (across the two passages there were 21 test words in this category; e.g., captive, snacks) and those with one or two other letters with the same ascending (or descending) feature (across the two passages there were 19 test words in this category; e.g., camping, talking). The s and c test words were not included in this analysis because all $\mathbf{4 0}$ of these words have at least one other letter with the neutral feature (recall that each test word included the neutral letter a). An ANOVA was conducted with subjects as the random effect and letter case and number of other letters with the same critical feature (zero or at least one) as the two within-subject factors. According to the alternative explanation, but not according to the explanation with respect to word shape, there should be a significant interaction of the two factors; subjects should make fewer proofreading errors in lowercase print when there are no other letters with the same critical feature than when there is at least one other letter with that feature. Although letter case yielded a significant main effect in this analysis $\left[F(1,39)=4.77, M S_{\mathrm{e}}=134.36, p=.033\right]$, reflecting the fact that more errors were made on uppercase than on lowercase, the number of other letters with the critical feature did not yield a significant main effect or a significant interaction with letter case $(F<1$ for each effect), contrary to the alternative explanation.

To make a broader assessment of the effects of age on proofreading errors, a mixed factor ANOVA was performed on the combined data of Experiments 1 and 2, with the between-subject factor of age level (Grade 4, Grade 7, college) and the within-subject factors of letter case and type of letter deletion. As in the analysis of Experiment 1 , there was a significant main effect due to age $\left[F(2,157)=24.95, M S_{c}=777.21, p<.001\right]$. The percentage of proofreading errors decreased markedly from $34.06 \%$ at Grade 4 , and $19.81 \%$ at Grade 7 , to $14.19 \%$ for the college students. The variable of case, significant in the analysis of Experiment 1 but not significant in the full analysis of Experiment 2, was marginally significant in the present analysis $\left[F(1,157)=3.35, M S_{\mathrm{e}}=68.28\right.$, $p=.069$ ].

The same pattern of proofreading errors found separately for Experiments 1 and 2 on misspelled $s$ and $c$ test words and $k$ and $p$ test words in lowercase and uppercase was present in this analysis combining Experiments 1 and 2; the interaction of letter case and type of letter deletion was significant $\left[F(1,157)=38.57, M S_{\mathrm{e}}=49.07\right.$, $p<.001]$. According to an $L S D$ test $(\alpha=.05, L S D=$ 1.55 ), the percentage of proofreading errors was significantly greater for the $s$ and $\mathrm{c}$ test words $(M=23.91 \%)$ than for the p and $\mathrm{k}$ test words $(M=20.28 \%)$ in lowercase, whereas the percentage of proofreading errors was significantly lower for the $\mathrm{s}$ and $\mathrm{c}$ test words $(M=$ $21.59 \%)$ than for the $\mathrm{k}$ and $\mathrm{p}$ test words in uppercase $(M$ $=24.94 \%$ ). Similarly, there was a significant increase in proofreading errors for lowercase relative to uppercase for the neutral $s$ and $c$ test words, whereas the opposite pattern was significant for the $k$ and $p$ test words. As illustrated in Figure 1, the overall performance of college students is remarkably similar to that of elementary school children when letter case and type of letter deletion are considered.

An additional mixed factor ANOVA was conducted on the combined data from Experiments 1 and 2 to determine whether the same pattern of results was evident in the two passages, which were seen by different subjects and included different test words (i.e., the two passages

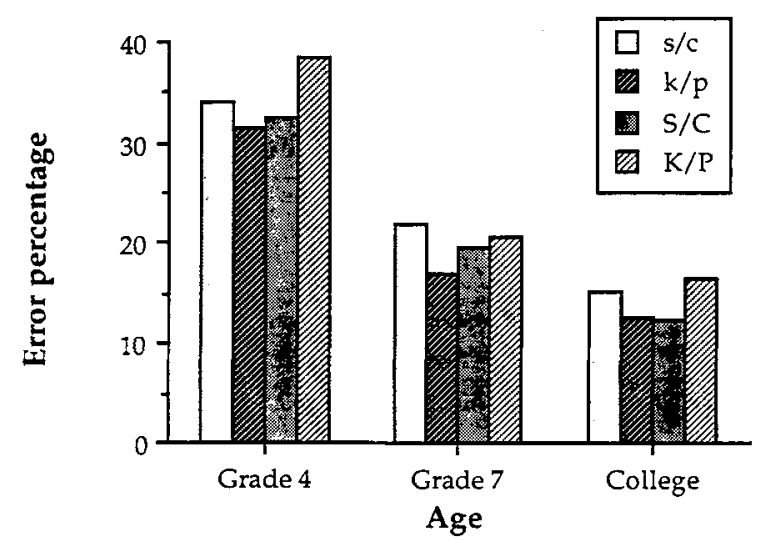

Figure 1. Percentages of errors made by fourth- and seventh-grade children and college students in proofreading lowercase and uppercase versions of a passage containing misspelled test words either with $s$ and $c$ or with $k$ and $p$ deleted in Experiments 1 and 2. 
had the same words, but a word which was misspelled in one passage was correctly spelled in the other passage). The ANOVA was performed with two between-subject factors (subject age and passage) and two within-subject factors (letter case and type of letter deletion). As in the previous analysis, there was a significant main effect of subject age $\left[F(2,154)=27.10, M S_{\mathrm{e}}=715.58, p<.001\right]$ and, importantly, a significant interaction of letter case and type of letter deletion $\left[F(1,154)=37.92, M S_{e}=\right.$ $49.91, p<.001]$. In addition, there was a significant main effect of passage $\left[F(1,154)=15.49, M S_{\mathrm{e}}=715.58\right.$, $p<.001]$, reflecting the fact that more errors were made on Passage 1 (26.89\%) than on Passage 2 (18.47\%), and a significant interaction between passage and type of letter deletion $\left[F(1,154)=40.23, M S_{e}=84.68, p<.001\right]$. (Although we had no a priori hypotheses concerning the conditions involved in this interaction, we decided to use the $L S D$ test to be consistent with our earlier tests of paired comparisons and because a Type I error in no way can be perceived as aiding our theoretical arguments.) According to an $L S D$ test $(\alpha=.05, L S D=2.91)$, this interaction was due to the fact that for Passage 1, more errors occurred on $s$ and $c$ words (29.34\%) than on $k$ and $p$ words (24.44\%), whereas for Passage 2, more errors occurred on $k$ and $p$ words $(20.78 \%)$ than on $s$ and $c$ words (16.16\%). Most crucially, however, there was no threeway interaction of letter case, passage, and type of letter deletion $[F(1,154)<1]$. For both passages (and for all ages), errors increased from uppercase to lowercase with the $s$ and $c$ words but decreased from uppercase to lowercase with the $p$ and $\mathbf{k}$ words. In other words, in every age and passage combination, the percentage of errors increased for the lowercase $s$ and $c$ deletions but decreased for the lowercase $p$ and $k$ deletions relative to the uppercase baselines. Thus, the percentage of proofreading errors did depend on the specific passages and the specific test words employed (i.e., the subjects understandably detected the misspellings on some words more easily than on other words), but both passages showed the predicted effects of word shape: Relative to the uppercase baseline, when the misspelling maintained the shape of the word in lowercase, proofreading error rates increased, but when the misspelling changed word shape in lowercase, proofreading error rates decreased.

Proofreading times. The mean time to proofread the two passages is shown in Table 3. An ANOVA was conducted on the combined proofreading times of the collegestudent subjects in Experiment 2 and those of the fourthand seventh-grade subjects in Experiment 1 . Only the single between-subject factor of age was included. The analysis indicated that the time needed to complete the proofreading task decreased as the age of the subjects increased $\left[F(2,156)=54.57, M S_{e}=36,552.57, p<.001\right]$. The mean reading times showed a marked decline from $897.6 \mathrm{sec}$ for Grade 4 to $676.7 \mathrm{sec}$ for Grade 7 and $471.5 \mathrm{sec}$ for the college students.

Comprehension test scores. The mean frequency of correct responses on the nine-item comprehension test is shown in Table 3. An ANOVA was performed on the combined comprehension scores for the college students in Experiment 2 and the fourth- and seventh-grade children in Experiment 1. It included the single betweensubject factor of age and indicated a decrease in score as age decreased from the college students $(M=8.5)$ to Grade $7(M=8.2)$ to Grade $4[M=7.9 ; F(2,157)=$ $4.58, M S_{e}=0.86, p=.012$, for all three age levels].

Spelling test. Only one error was made by one college student on the spelling test. Thus, the mean percentage of correct responses was $99.1 \%$ and the standard error of the mean was $0.1 \%$. As shown in Table 4, the single error was on a word with $\mathbf{s}$ or $c$ deleted.

\section{GENERAL DISCUSSION}

Our findings clearly support the position that word shape is an important variable in processing familiar words. When word shape is disrupted in lowercase print, word recognition is impeded, as reflected in the reduced number of proofreading errors made by our subjects on words in which ascending/descending letters were deleted.

These findings are inconsistent with the notion that word recognition is achieved solely by means of processing letters and letter features. The processing of word-shape supraletter features must be postulated to account for the differences between deleting letters of the two types (neutral and ascending/descending) because the deleted letters are not available to affect letter identification processes. (Note that the supraletter feature of word length was uniformly modified in all misspelled words, because with both typecases and with neutral letters, as well as with ascending and descending letters, deletions necessarily shorten the words.) Furthermore, the differences between the two types of deleted letters cannot be attributed to their location in the word, their pronunciation, the length or frequency of the words containing them, or any other linguistic variables not pertaining to their visual features, because the pattern of proofreading errors differs markedly for lowercase and uppercase print. Moreover, for each of the four critical letters, the visual features are highly similar in the two typecases except for the height features defining shape, so that only the shape variable is left to account for the particular pattern of proofreading errors made by the subjects.

To accommodate the present findings, an adequate model of word processing must acknowledge the contribution of global word-shape information. Therefore, models that currently do not include supraletter feature detection (e.g., McClelland \& Rumelhart, 1981) need to be modified or expanded. Although it thus seems mandatory to postulate the processing of word-shape information, it is not clear at what stage this processing $\alpha-$ curs. Perhaps supraletter features contribute to initial lexical access. Alternatively, such features may play a role at a subsequent stage of processing such as a verification stage (see Paap, Newsome, McDonald, \& Schvaneveldt, 1982) or a postperceptual cognitive-memorial stage (see Johnson, 1981). The present findings cannot discriminate between these possibilities. 
It is interesting that the effect of word shape did not vary as a function of age and reading level. Although this result was not expected based on the earlier work on word unitization (Cunningham et al., 1988; Drewnowski, 1978, 1981), it is consistent with the earlier findings from the picture-word interference task (Posnansky \& Rayner, 1977; but see Guttentag, 1981, who found word shape more important for poor readers than for good readers). This result may have been aided by the use of words that were familiar to subjects in all of our age groups, because the passage was originally constructed so that it could be understood by fourth-grade students. We interpret the lack of interactions between word shape and age or between word shape and reading level as an indication that word shape is a basic feature of written material that is processed by old and young readers of varying ability levels, given a minimum level of reading experience, that is, third- or fourth-grade level.

Although the effect of word shape was not influenced by the factors of age and reading level, these factors did significantly affect overall performance on the proofreading task, both with respect to speed and accuracy of proofreading and with respect to accuracy on the subsequent comprehension test. These findings substantiate the differences in reading ability among our various subject groups and demonstrate the sensitivity of the proofreading task to reading-ability level. The fact that the comprehension test scores were high overall (means ranging from $82.2 \%-95.6 \%$; see Table 3 ) further indicates that in performing the proofreading task, the subjects did indeed read the passage for meaning.

The high level of performance on the spelling test (only 77 out of 6,400 possible errors across all subjects) suggests that the proofreading errors made by the subjects were not due to the subjects' lack of spelling knowledge. The findings concerning the spelling test must, however, be interpreted with some caution because the correct answer on the test was always the longer of the two alternative spellings (misspellings were always created by deleting letters), and it is possible that the subjects became aware of this constraint, at least by the end of the test.

With this caveat in mind, we can ask why subjects who know how to spell a word might fail to detect a misspelling of it when proofreading. An obvious answer seems to be that when reading for meaning (even when proofreading for misspellings is required) subjects are able to identify words without identifying each of their component letters; they base their recognition decisions on partial information extracted from the word and do not require full information to be extracted before moving their attention to the following word. Just as it has been shown in other proofreading studies (e.g., Healy, 1981; Healy, Volbrecht, \& Nye, 1983) that subjects tolerate misspellings involving missing letter features (e.g., when $\mathrm{e}$ is replaced by c) as long as letter envelope is maintained, the present experiment indicates that subjects tolerate misspellings involving missing letters as long as word shape (and end letters) is maintained. More specifically, previous research (Healy, 1981; Healy et al., 1983) provided evidence that in searching for misspellings, proofreaders employ a specific pair of information-processing rules-namely, a hierarchical feature test (see, e.g., Lupker, 1979; Massaro \& Schmuller, 1975) coupled with a sophisticated-guessing decision rule (see, e.g., Broadbent, 1967; Lindsay \& Norman, 1977; Rumelhart \& Siple, 1974). These rules had been defined with respect to letter features but can easily be extended to supraletter features. In the hierarchical feature test as originally proposed, proofreaders give the greatest weight to resolving letter envelope and give less weight to resolving other letter features. In the extended version of the hierarchical feature test, proofreaders would give the greatest weight to resolving word shape and less weight to resolving supraletter features, such as word length, that do not contribute to word shape. Similarly, in the sophisticated-guessing decision rule as originally proposed, when letter envelope conforms to expectations, proofreaders tolerate the absence of letter features but are intolerant of additional features. In the extended version of this rule, when word shape conforms to expectations, proofreaders would tolerate the absence of letters but would be intolerant of added letters. Although misspellings involving added letters were not included in the present experiment, Monk and Hulme (1983) compared misspellings created by substituting and deleting letters and found that proofreaders indeed missed deletions more often than substitutions.

The present findings are also consistent with the work on the letter detection task supporting a unitization model of reading (see, e.g., Healy, Conboy, \& Drewnowski, 1987; Healy \& Drewnowski, 1983; Healy, Oliver, \& McNamara, 1987). This work provides evidence both for the existence of reading units larger than the letter and for the importance of supraletter features in word processing. More specifically, a "missing letter" effect has been obtained (see, e.g., Drewnowski \& Healy, 1977) when subjects are asked to read a passage of text and circle every instance of a given target letter (e.g., the letter $t$ ). The missing letter effect refers to the finding of a greater number of letter detection errors on highly familiar words, such as the, than on rare words, such as thy (Healy, 1976, 1980). The inference that is drawn from this effect is that highly familiar words are often processed at the wordunit level without completed processing at the letter level. One criticism of these findings (see Oden, 1984) is that the evidence for word-unit processing comes largely from common function words such as the and and. The present findings concerning the processing of word-shape information are not subject to this criticism because the crucial test words in the present experiments were less familiar content words. Indeed, the present experiments provide an extremely conservative test of the importance of word shape because all of the crucial test words were at least four letters long, and previous investigators (e.g., R. N. Haber \& Schindler, 1981) have found the largest effects of word shape with short words.

Although the present study provides clear evidence for the importance of word shape to word processing, the precise definition of word shape is still open to question. The 
simple definition we used, based on Bouma's (1971) classification of lowercase letters into three categories, was able to provide an account of the present set of findings. However, a more complete definition of shape may be needed to account for the results of further investigations. For example, a fuller definition might distinguish between different uppercase letters, might include the variables of height-to-width quotient (see Bouma, 1971) and letter density (see R. N. Haber \& L. R. Haber, 1981), and might allow for word length to make some contribution to word shape, especially when the length difference between two words is substantial (e.g., compare the shapes of hassock and hat). Also, the relevant level of processing needs to be determined. Although we, along with other investigators, refer to word shape as the relevant variable, our results are equally consistent with the notion that syllable shape, for example, is the crucial variable. It is clear from our study that supraletter features influence word recognition, but the nature and extent of those features needs to be specified in further research.

\section{REFERENCES}

Aвramovici, S. (1983). Errors in proofreading: Evidence for syntactic control of letter processing? Memory \& Cognition, 11, 258-261. BESNER, D. (1989). On the role of outline shape and word-specific visual pattern in the identification of function words: None. Quarterly Joumal of Experimental Psychology, 41A, 91-105.

BoumA, H. (1971). Visual recognition of isolated lower-case letters. Vision Research, 11, 459-474.

BrondBent, D. E. (1967). Word-frequency effect and response bias. Psychological Review, 74, 1-15.

CAtTEl, J. M. (1886). The time taken up by cerebral operations. Mind, 11, 220-242, 377-392, 524-538.

Corcoran, D. W. J. (1967). Acoustic factor in proof reading. Nature, 214, $851-852$

Cunningham, T. F., Healy, A. F., Kanengiser, N., Chizzick, L., * Wilutrts, R. L. (1988). Investigating the boundaries of reading units across ages and reading levels. Joumal of Experimental Child Psychology, 45, 175-208.

DrewnowskI, A. (1978). Detection errors on the word the: Evidence for the acquisition of reading levels. Memory \& Cognition, 6, 403-409.

DrewnowsKI, A. (1981). Missing -ing in reading: Developmental changes in reading units. Joumal of Experimental Child Psychology, 31, 154-168.

Drewnowski, A., Healy, A. F. (1977). Detection errors on the and and: Evidence for reading units larger than the word. Memory \& Cognition, 5, 636-647.

ERdmann, B., \& Dodge, R. (1898). Psychologische untersuchungen uber das lesen, auf experimetaller grundlage [Psychological investigations of reading, on an experimental basis]. Halle, Germany: Niemeyer.

GarNer, W. R. (1981). The role of configuration in the identification of visually degraded words. Memory \& Cognition, 9, 445-452.

Guttentag, R. E. (1981). The role of word shape as a recognition cue in children's automatic word processing. Child Development, 52 , 363-366.

Haber, L. R., Haber, R. N., \& Furdin, K. R. (1983). Word length and word shape as sources of information in reading. Reading Research Quarterly, 18, 165-189.

HABER, R. N., HABer, L. R. (1981). Visual components of the reading process. Visible Language, 15, 147-182.

HABER, R. N., SCHINDLER, R. M. (1981). Error in proofreading: Evidence of syntactic control of letter processing? Journal of Experimental Psychology: Human Perception \& Performance, 7, 573-579.

HADley, J. A., \& HeALY, A. F. (1991). When are reading units larger than the letter? Refinement of the unitization reading model. Journal of Experimental Psychology: Leaming, Memory, \& Cognition, 17, 1062-1073.

HeAly, A. F. (1976). Detection errors on the word the: Evidence for reading units larger than letters. Journal of Experimental Psychology: Human Perception \& Performance, 2, 235-242.

Healy, A. F. (1980). Proofreading errors on the word the: New evidence on reading units. Journal of Experimental Psychology: Human Perception \& Performance, 6, 45-57.

Healy, A. F. (1981). The effects of visual similarity on proofreading for misspellings. Memory \& Cognition, 9, 453-460.

Healy, A. F., Conboy, G. L., \& Drewnowski, A. (1987). Characterizing the processing units of reading: Effects of intra- and interword spaces in a letter detection task. In B. K. Britton \& S. M. Glynn (Eds.), Executive control processes in reading (pp. 279-296). Hillsdale, NJ: Erlbaum.

HeAly, A. F., DrewnowsKa, A. (1983). Investigating the boundaries of reading units: Letter detection in misspelled words. Joumal of Experimental Psychology: Human Perception \& Performance, 9, 413-426.

Healy, A. F., Olver, W. L., McNamara, T. P. (1987). Detecting letters in continuous text: Effects of display size. Joumal of Experimental Psychology: Human Perception \& Performance, 13, 279-290.

Healy, A. F., Volbrecht, V. J., * Nye, T. R. (1983). The effects of perceptual condition on proofreading for misspellings. Memory \& Cognition, 11, 528-538.

JohNSON, N. F. (1981). Integration processes in word recognition. In O. J. L. Tzeng \& H. Singer (Eds.), Perception of print: Reading research in experimental psychology (pp. 29-63). Hillsdale, NJ: Erlbaum.

Lindsay, P. H., Norman, D. A. (1977). Human information processing: An introduction to psychology (2nd ed.). New York: Academic Press.

LUPKER, S. J. (1979). On the nature of perceptual information during letter perception. Perception \& Psychophysics, 25, 303-312.

Massaro, D. W., Schmuller, J. (1975). Visual features, preperceptual storage, and processing time in reading. In D. W. Massaro (Ed.), Understanding language: An information processing analysis of speech perception, reading, and psycholinguistics (pp. 207-239). New York: Academic Press.

MCCLELLAND, J. L. (1977). Letter and configuration information in word identification. Journal of Verbal Learning \& Verbal Behavior, 16, $137-150$

McClelland, J. L., Rumelhart, D. E. (1981). An interactive activation model of context effects in letter perception: Part 1 . An account of basic findings. Psychological Review, 88, 375-407.

Monk, A. F., Hulme, C. (1983). Errors in proofreading: Evidence for the use of word shape in word recognition. Memory \& Cognition, 11, 16-23.

ODEN, G. C. (1984). Dependence, independence, and emergence of word features. Joumal of Experimental Psychology: Human Perception \& Performance, 10, 394-405

O'REGAN, K. (1979). Saccade size control in reading: Evidence for the linguistic control hypothesis. Perception \& Psychophysics, 25, $501-509$

PaAp, K. R., Newsome, S. L., McDonald, J. E., Schyaneveldt, R. W. (1982). An activation-verification model for letter and word recognition: The word-superiority effect. Psychological Review, 89, 573-594.

PaAp, K. R., Newsome, S. L., \& Noel, R. W. (1984). Word shape's in poor shape for the race to the lexicon. Journal of Experimental Psychology: Human Perception \& Performance, 10, 413-428.

Posnansky, C. J., \& RAyner, K. (1977). Visual-feature and response components in a picture-word interference task with beginning and skilled readers. Journal of Experimental Child Psychology, 24, $440-460$.

RAYNER, K. (1979). Eye guidance in reading: Fixation locations within words. Perception, 8, 21-30.

RAYNER, K., MCConkIE, G. W., Z Zola, D. (1980). Integrating information across eye movements. Cognitive Psychology, 12, 206-226. Reading test for New York State elementary schools: Grade 6-Form E manual for administrators and teachers (1982). Albany, NY: University of the State of New York, State Education Department. 
Reading test for New York State elementary schools: Grade 3-Form R manual for administrators and teachers (1983). Albany, NY: University of the State of New York, State Education Department.

Rumelhart, D. E., Siple, P. (1974). Process of recognizing tachistoscopically presented words. Psychological Review, 81, 99-113.

STroop, J. R. (1935). Studies of interference in serial verbal reactions. Journal of Experimental Psychology, 18, 643-662.

UNDERWOOD, G., BARGH, K. (1982). Word shape, orthographic regularity, and contextual interactions in a reading task. Cognition, 12, 197-209.

WALKER, P. (1987). Word shape as a cue to the identity of a word: An analysis of the Kučera and Francis (1967) word list. Quarterly Journal of Experimental Psychology, 39A, 675-700.

\section{NOTE}

1. Although only four letters were selected for this purpose, we essentially exhausted the set of letters that could be used, because most other letters have different features in lowercase and uppercase print. The only other letters with the same features in the two typecases either are vowels (o and $u$ ) or occur with low frequency in English $(v, w$, $x$, and $z$ ). The four letters we did employ are all consonants, and all occur in English with relatively high frequency.

\section{APPENDIX}

Test Words in Each Passage With Error Frequencies in Experiment 2 as a Function of Letter Cace

\begin{tabular}{|c|c|c|}
\hline \multicolumn{3}{|c|}{$\begin{array}{c}\text { Passage } 1 \\
\text { Words with } p\end{array}$} \\
\hline camping & 1 & CAMPING \\
\hline newspaper & 9 & NEWSPAPER \\
\hline champion & 1 & CHAMPION \\
\hline clasped & 0 & CLASPED \\
\hline rapid̄ly & 8 & RAPIDLY \\
\hline captive & 6 & CAPTIVE \\
\hline sharpened & 4 & SHARPENED \\
\hline sample & 4 & SAMPLE \\
\hline snapshot & 3 & SNAPSHOT \\
\hline caphre & 5 & CAPTURE \\
\hline \multicolumn{3}{|c|}{ Words with $k$} \\
\hline sneakers & 4 & SNEAKERS \\
\hline market & 2 & MARKET \\
\hline sweetcakes & 2 & SWEETCAKES \\
\hline breaking & 0 & BREAKING \\
\hline parking & 1 & PARKIING \\
\hline bakery & 0 & BAKEERY \\
\hline sparkle & 0 & SPARKLE \\
\hline tanker & 9 & TANEEER \\
\hline mistake & 0 & MISTAKEE \\
\hline shaking & 2 & SHAKING \\
\hline \multicolumn{3}{|c|}{ Words with s } \\
\hline disaster & 12 & DISASTER \\
\hline smashed & 1 & SMASWHED \\
\hline raișins & 0 & RAISINS \\
\hline case & 1 & CASEE \\
\hline hamster & 0 & HAMSTER \\
\hline flash & 0 & FLASSH \\
\hline fastened & 3 & FASTTENED \\
\hline vanish & 1 & VANISIH \\
\hline bashed & 7 & BASHED \\
\hline taste & 1 & TASTE \\
\hline \multicolumn{3}{|c|}{ Words with $\mathrm{c}$} \\
\hline $\begin{array}{l}\text { laçing } \\
\text { fractured }\end{array}$ & $\begin{array}{l}2 \\
2\end{array}$ & $\begin{array}{l}\text { LACING } \\
\text { FRACTURED }\end{array}$ \\
\hline
\end{tabular}

APPENDIX (Continued)

$\begin{array}{lrlr}\text { packed } & 8 & \text { PACKED } & 7 \\ \text { catch } & 10 & \text { CATCH } & 5 \\ \text { scratched } & 14 & \text { SCRATCHED } & 9 \\ \text { backdoor } & 0 & \text { BACKDOOR } & 0 \\ \text { reaction } & 12 & \text { REACTION } & 11 \\ \text { watched } & 8 & \text { WATCHED } & 5 \\ \text { chance } & 6 & \text { CHANCEE } & 4 \\ \text { sauce } & 0 & \text { SAUCE } & 0\end{array}$

Passage 2

Words with $\mathrm{p}$

$\begin{array}{ll}\text { collapsed } & 4 \\ \text { grapefruit } & 4 \\ \text { carpet } & 3 \\ \text { cheaper } & 0 \\ \text { napkin } & 1 \\ \text { damper } & 1 \\ \text { trampled } & 3 \\ \text { escaped } & 3 \\ \text { grasped } & 8 \\ \text { scrapbook } & 4\end{array}$

$\begin{array}{ll}\text { COLLAPSED } & 5 \\ \text { GRAPEFRUIT } & 6 \\ \text { CARPET } & 7 \\ \text { CHEAPER } & 1 \\ \text { NAPKIN } & 0 \\ \text { DAMPER } & 2 \\ \text { TRAMPLED } & 5 \\ \text { ESCAPED } & 4 \\ \text { GRASPED } & 6 \\ \text { SCRAPBOOK } & 6\end{array}$

Words with $k$

snacks

SNACKS

CAKE

dashed

task cake

make

basket

snake

talking

breakers

raked

darken

walked

MAKE

BASKET

SNAKE

TALKJNG

BREAKERS

RAKED

DARKEN

WALKED

wash

cash

plastic

because

chasing

laste

crassh

casping

DASHED

WASYM

CAST

TASKK

PLASTIC

BECAUŞE

CHASING

LAST

CRASH

CASTING

beach

bascon

space

jacket

acklebox

$C$

Words with

BEACH

BACON

SPACE

JACKET

TACKLEBOX

PACE

RACING

PLACE

YACHT

CRACK

crack 2

Note-Deleted letter is underlined.

0
1
0
3
3
2
6

0

0

I

8

0

3

0

place

(1)

